\title{
O VALOR DOS NÚMEROS ${ }^{1}$
}

\section{TESE}

PEDREIRA, Sinvaldo Martins ${ }^{2}$

PEDREIRA, Sinvaldo Martins. O valor dos números. Revista Científica Multidisciplinar Núcleo do Conhecimento. Ano 01, Vol. 08. pp. 05-16. Setembro de 2016. ISSN: 2448-0959, Link de acesso: https://www.nucleodoconhecimento.com.br/matematica/o-valor-dos-numeros, DOI: 10.32749/nucleodoconhecimento.com.br/matematica/o-valor-dos-numeros

\section{RESUMO}

Este trabalho tem por objetivo mostrar uma nova visão sobre o que são os números e sua representatividade junto a realidade, através de uma sistemática objetiva e racional, onde se possam caracterizar as etapas equacionais de forma clara e sucinta, corrigindo anomalias aritméticas causadas por equívocos de interpretação de como se comportam os termos numéricos, haja visto que são comumente utilizados no contexto matemático e nas diferentes unidades fatoriais de forma generalizada, sem se preocupar com a singularidade individual que cada componente possui.

Ao longo da história humana o homem tem seguido de forma catedrática as regras impostas pelo sistema vigente e quando se encontra em uma encruzilhada inventa regras mirabolantes, de alto grau de criatividade, porém de pouca eficácia já que suas soluções se encontram no plano do imaginário não no plano da realidade, se baseiam puramente em regras, não em preceitos racionais.

\footnotetext{
${ }^{1}$ Este trabalho trata-se de resultado das pesquisas para desenvolvimento de uma tese sobre o comportamento entre números positivos e negativo, dando embasamento para que o autor recriasse uma teoria paralela.

${ }^{2}$ Matemático, Funcionário Público da Cidade de São Paulo.
}

RC: 4359

Disponível em: https://www.nucleodoconhecimento.com.br/matematica/o-valor-dosnumeros 


\section{PREFÁCIO}

Os números foram criados para quantificar algo, seja pela proporção ou medida (comprimento, área, volume, tempo, peso, etc....).

Mundialmente convencionou-se o uso dos símbolos numéricos arábicos $(0,1,2,3$, $4,5,6,7,8,9)$

Onde:

O símbolo $\underline{0}$ identifica o número zero.

O símbolo 1 identifica o número um.

O símbolo 2 identifica o número dois.

O símbolo $\underline{3}$ identifica o número três.

E aí por diante...

Também ficou convencionado que seriam agrupados em unidades, dezenas, centenas, etc....

Ex:

123: cento e vinte e três.

Uma centena, duas dezenas e três unidades.

De posse de um preceito matemático as pessoas aprenderam a contar as coisas, soma-las e subtrai-las.

*Obs. Com o advento da subtração passaram a existir os números negativos, identificados pelo sinal (-) antes do número.

RC: 4359

Disponível em: https://www.nucleodoconhecimento.com.br/matematica/o-valor-dosnumeros 
Ex:

$-1=$ menos um

$-15=$ menos quinze

E os números ficaram ordenados da seguinte forma:

$(\ldots-6,-5,-4,-3,-2,-1,0,1,2,3,4,5,6 \ldots)$

Posteriormente aprendeu-se a multiplicar e dividir as coisas, criando assim, as quatro equações fundamentais.

Adição (+).

Subtração (-).

Multiplicação (x).

Divisão (/).

Adição: $5+6=11 \quad 12+3=15 \quad 4+8=12 \quad 13+18=31$

Subtração: $8-3=5 \quad 15-4=11 \quad 25-7=18 \quad 20-6=14$

Multiplicação: $7 \times 5=35 \quad 2 \times 4=8 \quad 6 \times 9=54 \quad 12 \times 3=36$

Divisão: $8 / 2=4 \quad 40 / 5=8 \quad 70 / 10=7 \quad 55 / 11=5$

Porém notaram-se algumas peculiaridades quando usamos números negativos (-), não somente nas subtrações, mas de forma diversas:

\section{1ำ peculiaridade:}

Subtração: $-4+3=-1 \quad-7+8=+1 \quad-15+8=-7 \quad-2+1=-1$ 
Adição: $\quad+8+6=+14 \quad+1+2=+3 \quad-5-5=-10 \quad-12-13=-25$

Multiplicação: $+3 \times 7=+21 \quad+3 \times-7=-21 \quad-3 \times 7=-21 \quad-3 \times-7=+21$

Divisão: $\quad+10 /+5=+2 \quad+10 /-5=-2 \quad-10 /+5=-2 \quad-10 /-5=+2$

Quando um número é multiplicado ou dividido a regra é a seguinte:

(Mais com mais) $++=+$ (positivo)

(Mais com menos) $+-=-$ (negativo)

(Menos com mais) $-+=-$ (negativo)

(Menos com menos) - - = + (positivo)

\section{0 peculiaridade}

\section{Potenciação e radiciação.}

$+5^{2}=25 \quad-5^{2}=+25 \quad+5^{3}=+125 \quad-5^{3}=-125$

$+3^{2}=9 \quad-3^{2}=+9 \quad+3^{3}=+27 \quad-3^{3}=-27$

Seguindo a regra.

$+5 x+5=+25 \quad-5 x-5=+25 \quad+5 x+5 x+5=+125 \quad-5 x-5 x-5=-125$

$+3 x+3=+9 \quad-3 x-3=+9 \quad+3 x+3 x+3=+27 \quad-3 x-3 x-3=-27$

Correspondente:

\begin{tabular}{lllllllll}
-125 & -25 & -5 & -1 & 0 & 1 & 5 & 25 & 125 \\
\hline
\end{tabular}

$5^{2} \quad 5^{3}$

RC: 4359

Disponível em: https://www.nucleodoconhecimento.com.br/matematica/o-valor-dosnumeros 
Agrupamento

$+5^{\star}=1,5,25,125, \ldots .$. ordenado

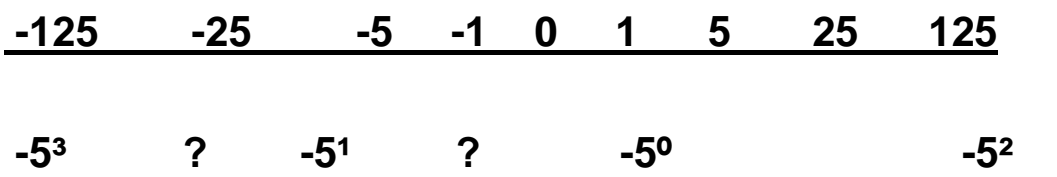

Agrupamento

$-5^{\star}=1,-5,25,-125, \ldots . .$. aleatório

\begin{tabular}{ccccccccc}
-27 & -9 & -3 & -1 & 0 & 1 & 3 & 9 & 27 \\
\hline $3^{\circ}$ & $3^{1}$ & $3^{2}$ & $3^{3}$ & & & & &
\end{tabular}

Agrupamento

$+3^{*}=1,3,9,27, \ldots \ldots$ ordenado

$\begin{array}{ccccccccc}-27 & -9 & -3 & -1 & 0 & 1 & 3 & 9 & 27 \\ & & & & & & & & \\ & -3^{3} & ? & -3^{1} & ? & & 30 & -3^{2}\end{array}$

Agrupamento

$-3^{*}=1,-3,9,-27, \ldots .$. aleatório

\section{3o peculiaridade}

RC: 4359

Disponível em: https://www.nucleodoconhecimento.com.br/matematica/o-valor-dosnumeros 
$2+0=2$

$2-0=2$

$2 \times 0=0$

2 / 0 = não existe resposta satisfatória (incógnita).

\section{O VALOR DOS NÚMEROS}

Até o momento vimos que os números surgiram para quantificar as coisas, sendo criadas para isso as quatro equações fundamentais:

Adição, subtração, multiplicação e divisão.

Vimos também que os números são representados por dois sinais distintos, (+) positivos e (-) negativos, também sendo planificados desta forma:

$\ldots-10-9-8-7-6-5-4-3-2-1 \quad 0+1+2+3+4+5+6+7+8+9+10 \ldots$

\section{COMO SÃO UTILIZADOS}

Ao trabalharmos com cálculos tratamos os termos de forma genérica, são positivos (+) ou negativos (-), sem nos preocupar com o que cada termo numérico representa.

Ex:

$+50-30=20+50+30=80$

$-50-30=-80-50+30=-20$

$+50-(-30)=+80 \quad+50+(-30)=+20$

RC: 4359

Disponível em: https://www.nucleodoconhecimento.com.br/matematica/o-valor-dosnumeros 
$+50+(+30)=+80 \quad-50-(-30)=-20$

$-50+(+30)=-20 \quad-50-(+30)=-80$

$5 \times 3=15 \quad 5 \times-3=-15$

$-5 \times 3=-15 \quad-5 \times-3=+15$

$10 / 2=+5 \quad 10 /-2=-5$

$-10 / 2=-5 \quad-10 /-2=+5$

Todos os cálculos estão corretos segundo as regras vigentes.

Será que estão corretos mesmo?

\section{A RAIZ DA QUESTÃO}

Para uma melhor visualização da questão, vamos chamar os números:

Positivos (+) de "Concretos".

Por que concretos $(+)$ ?

Porque representam algo, alguma coisa existente.

Negativos (-) de "Abstratos".

Por que abstratos (-)?

Porque representam a falta de algo, uma pendência.

RC: 4359

Disponível em: https://www.nucleodoconhecimento.com.br/matematica/o-valor-dosnumeros 


\section{OBSERVAÇÃO IMPORTANTE}

Foi convencionado o uso do sinal (+) tanto para indicar que um numero é positivo como para indicar uma adição (soma), porem isso é um equívoco aritmético, já que a condição de um número ser positivo indica uma qualidade numérica, enquanto que uma adição indica uma equação de união entre termos ou seja, uma ação entre fatores numéricos.

Foi convencionado o uso do sinal (-) tanto para indicar que um numero é negativo como para indicar uma subtração (diferença), porem isso é um equívoco aritmético, já que a condição de um número ser negativo indica uma qualidade numérica, enquanto que uma subtração indica uma equação de diferença entre termos ou seja, uma ação entre fatores numéricos.

Para resolver o problema:

Continuaremos utilizando o sinal (+) para identificar que o número é positivo e o sinal

(-) para identificar que o número é negativo.

A adição será representada pela letra $(U)$.

A subtração será representada pela letra $(\mathrm{H})$.

Ex:

$\begin{array}{lll}+5 \underline{U}+3=+8 & +7 \underline{U}+7=+14 & +4 \underline{U}+8=+12 \\ +3 \underline{U}-9=-6 & +5 \underline{U}-1=+4 & -8 \underline{U}-9=-17 \\ +9 \underline{\mathrm{H}}+5=+4 & +8 \underline{\mathrm{H}}-5=+13 & -7 \underline{\mathrm{H}}-8=+1 \\ -4 \underline{\mathrm{H}}-2=-2 & -2 \underline{\mathrm{H}}-4=+2 & +1 \underline{\mathrm{H}}-5=+6\end{array}$

RC: 4359

Disponível em: https://www.nucleodoconhecimento.com.br/matematica/o-valor-dosnumeros 


\section{PARADOXO}

\section{Multiplicação}

$+5 x+4=+20 \quad$ será?

+5 laranjas $\mathbf{x}+4$ laranjas = ? (Irracional) "coisas não multiplicam coisas".

$+5 \times-4=-20$ será?

+5 laranjas x -4 laranjas = ? (Irracional) "coisas não multiplicam pendências".

$-5 x+4=-20 \quad$ será?

-5 laranjas $\mathbf{x}+4$ laranjas = ? (Irracional) "pendências não multiplicam coisas".

$-5 \times-4=+20 \quad$ será?

-5 laranjas x -4 laranjas = ? (Irracional) "pendências não multiplicam pendências".

\section{Divisão}

$+10 /+2=+5$ será?

+10 pedras / +2 pedras = ? (Irracional) "coisas não são divididas por coisas".

$+10 /-2=-5$ será?

+10 pedras / -2 pedras = ? (Irracional) "coisas não são divididas por pendências".

$-10 /+2=-5 \quad$ será?

-10 pedras / +2 pedras = ? (Irracional) "pendências não são divididas por coisas".

$-10 /-2=-5 \quad$ será?

RC: 4359

Disponível em: https://www.nucleodoconhecimento.com.br/matematica/o-valor-dosnumeros 
-10 pedras / -2 pedras = ? (Irracional) "pendências não são divididas por pendências".

\section{Como resolver o problema?}

Para resolver o problema precisamos de um termo de valor neutro $\left({ }^{\circ} n\right)$, não sendo (+) concreto e nem (-) abstrato, um termo que represente a razão geométrica pela qual devemos multiplicar ou dividir as coisas ou pendências.

Para uma melhor visualização, vamos usar números neutros e chamaremos de:

$\left({ }^{\circ} \mathrm{n}\right)$. Geométricos, $(\mathrm{x})$ ou $(/)$. "Representam a razão geométrica pela qual um número deve ser multiplicado ou dividido, não tendo valor concreto ou abstrato, sendo então neutro".

Ex:

\section{Multiplicação}

$=8 x+5=+40$ será?

-8 $x+5$ laranjas $=+40$ laranjas (racional).

-8 $\times-5=-40$ será?

$98 \times-5$ laranjas $=-40$ laranjas (racional).

\section{Divisão}

$+10 / \stackrel{2}{2}=+5 \quad$ será?

+10 pedras $/ \stackrel{\circ}{2}=+5$ pedras (racional).

$-10 / \stackrel{2}{-2}=-5 \quad$ será?

RC: 4359

Disponível em: https://www.nucleodoconhecimento.com.br/matematica/o-valor-dosnumeros 
-10 pedras $/ \stackrel{0}{2}=-5$ pedras (racional).

\section{COMO DEVEM SER UTILIZADOS}

Tal qual foi visto, os termos de um cálculo não devem ser utilizados de forma genérica, apenas tendo valor (+) ou (-), devemos utilizar os termos conforme sua representatividade específica, para casos específicos.

Utilizaremos os símbolos:

(+) termo positivo (concreto)

(-) termo negativo (abstrato)

(ํnn) termo neutro (geométrico) usado em (x) multiplicação ou (/) divisão.

Os números possuem valor definidos ficando assim planificados:

$\mathbf{X}:$

$\stackrel{0}{6}(\mathrm{x})$

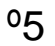

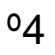

03

${ }^{\circ} 2$

01

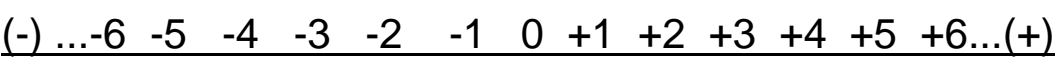

RC: 4359

Disponível em: https://www.nucleodoconhecimento.com.br/matematica/o-valor-dosnumeros 
01

02

03

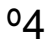

05

o6 (/)

I

No eixo das "Ordenadas "figuram somente os números $\left({ }^{\circ} n\right)$ Geométricos, sendo (x) multiplicação na parte superior e (/) divisão na parte inferior.

No eixo da "Abscissas "figuram os números (-) negativos do lado esquerdo e (+) positivos do lado direito.

\section{REGRAS}

Números (+) positivos e (-) negativos podem somar, subtrair, serem multiplicados ou divididos.

*Jamais multiplicar ou dividir.

Números $\left({ }^{\circ} n\right)$ geométricos, $(x)$ multiplicam ou (/) dividem números (+) positivos ou (-) abstratos.

*Jamais sendo multiplicados ou divididos por números (+) ou (-).

Ex:

RC: 4359

Disponível em: https://www.nucleodoconhecimento.com.br/matematica/o-valor-dosnumeros 
$+5 U+5=+10 \quad+5 U-6=-1 \quad-8 U+2=-6$

$-3 \cup-4=-7$

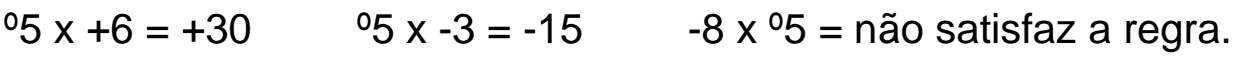

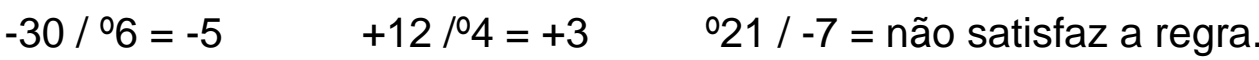

\section{Potenciacão}

$+5^{2}=25 \quad-5^{2}=-25 \quad+5^{3}=+125 \quad-5^{3}=-125$

$+3^{2}=9 \quad-3^{2}=-9 \quad+3^{3}=+27 \quad-3^{3}=-27$

\section{Seguindo a regra.}

$05 x+5=+25 \quad 05 x-5=-25 \quad 95 \times(-5 x+5)=+125$

$05 \times(-5 \times-5)=-125$

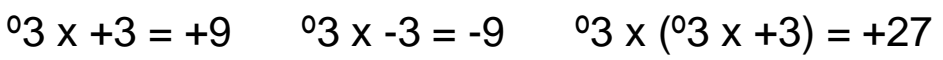

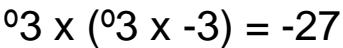

Correspondente:

\begin{tabular}{lllllllll}
-125 & -25 & -5 & -1 & 0 & 1 & 5 & 25 & 125 \\
\hline
\end{tabular}

$5^{\circ} \quad 5^{1} \quad 5^{2} \quad 5^{3}$

Agrupamento

RC: 4359

Disponível em: https://www.nucleodoconhecimento.com.br/matematica/o-valor-dosnumeros 
$+5^{\star}=1,5,25,125, \ldots \ldots$. Ordenado

$\begin{array}{lllllllll}-125 & -25 & -5 & -1 & 0 & 1 & 5 & 25 & 125-\end{array}$

$5^{3} \quad-5^{2} \quad-5^{1} \quad-5^{\circ}$

Agrupamento

$-5^{\star}=-1,-5,-25,-125, \ldots$ Ordenado

\begin{tabular}{lllllllll}
-27 & -9 & -3 & -1 & 0 & 1 & 3 & 9 & 27 \\
\hline
\end{tabular}

$\begin{array}{llll}3^{0} & 3^{1} & 3^{2} & 3^{3}\end{array}$

Agrupamento

$+3^{\star}=+1,+3,+9,+27, \ldots \ldots$ ordenado

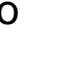




\section{CASO ZERO}

O número (0) zero é um termo insubstancial, não possui valor algum, representa o vazio, pois é nulo, não se confunda nulo com neutro.

O termo (0) zero não possui valor (+) positivo ou (-) negativo, não podendo ser adicionado ou subtraído, multiplicado ou dividido e como também não é ( $\left.{ }^{\circ} n\right)$ neutro não (x) multiplica ou (/) divide qualquer outro número.

O (0) zero serve única e exclusivamente com resultado de uma adição ou subtração onde os termos se anulam.

Ex:

$+4-4=0 \quad-8+3+5=0 \quad+35-10-25=0$

+2 +0 = não existe" (0) zero é nulo"

+2 -0 = não existe" (0) zero é nulo"

๑2 x 0 = não existe" (0) zero é nulo"

$0 /{ }^{\circ} 2$ = não existe" (0) zero é nulo"

\section{CASOS EXCEPCIONAIS}

Existem casos conceituais que devido ao fato de representarem medidas e não coisas, podem ser representados por números (+) positivos, (-) negativos e $\left({ }^{\circ} n\right)$ geométricos.

1․ Caso. Tempo: tanto pode se mover no eixo das Abscissas, quando subtrai ou adiciona tempo, como multiplicada e dividida.

RC: 4359

Disponível em: https://www.nucleodoconhecimento.com.br/matematica/o-valor-dosnumeros 
Abscissas com ordenadas, quando expressa aceleração.

2 caso. Dimensão: tanto pode se mover no eixo das Abscissas, quando subtrai ou adiciona medidas, como multiplicada e dividida.

Abscissas com ordenadas; quando expressa espaço.

Abscissas com ordenadas e profundidade; quando expressa dimensão.

3o caso. Massa: tanto pode se mover no eixo das Abscissas, quando exemplifica peso, podendo ser somada, subtraída, como multiplicada e dividida.

$4^{\circ}$ caso. Conceitos Físicos: os conceitos podem se fundir

ex: velocidade, densidade, força, etc...transitando de forma sistêmica, pelo eixo das Ordenadas, Abscissas e profundidade.

\section{CONSIDERAÇÕES FINAIS}

De posse de uma nova realidade matemática, abrem-se diversas possibilidades de resolvermos problemas nos mais diversos campos da ciência, tecnologia e racionalidade, já que as equações passarão a serem vistas de forma mais ampla, pois suas partes têm significado específico e próprio, um universo geométrico e tridimensional se formara de forma mais visível aos olhos de quem prestar atenção ao seu redor o limite é o infinito.

RC: 4359

Disponível em: https://www.nucleodoconhecimento.com.br/matematica/o-valor-dosnumeros 